\title{
Validity of the Canadian Paediatric Triage and Acuity Scale in a tertiary care hospital
}

\author{
Jocelyn Gravel, MD, MSc; Sergio Manzano, MD; Michael Arsenault, MD
}

\begin{abstract}
Objective: We evaluated the validity of the Canadian Paediatric Triage and Acuity Scale (PaedCTAS) for children visiting a pediatric emergency department (ED).

Methods: This was a retrospective study evaluating all children who presented to a pediatric university-affiliated ED during a 1-year period. Data were retrieved from the ED database. Information regarding triage and disposition was registered in an ED database by a clerk following patient management. In the absence of a gold standard for triage, admission to hospital, admission to pediatric intensive care unit (PICU) and length of stay (LOS) in the ED were used as surrogate markers of severity. The primary outcome measure was the correlation between triage level (from 1 to 5) and admission to hospital. The correlation between triage level and dichotomous outcomes was evaluated by a $\chi^{2}$ test and an analysis of variance (ANOVA) was used to evaluate the association between triage level and ED LOS.

Results: Over the 1-year period, 58529 patients were triaged in the ED. The proportion admitted to hospital was $63 \%$ for resuscitation (level 1), 37\% for emergent (level 2), 14\% for urgent (level 3), $2 \%$ for semiurgent (level 4) and $1 \%$ for nonurgent (level 5$)(p<0.001)$. There was also a good correlation between triage levels and LOS and admission to PICU (both $p<0.001$ ).

Conclusion: This computerized version of PaedCTAS demonstrates a strong association with admission to hospital, admission to PICU and LOS in the ED. These results suggest that PaedCTAS is a valid tool for triage of children in a pediatric ED.
\end{abstract}

Keywords: triage, pediatric, validity

\begin{abstract}
RÉSUMÉ
Objectif : Nous avons évalué la validité de l'Échelle canadienne de triage et de gravité (ÉTG) applicable aux enfants qui se présentent à un service d'urgence pédiatrique.

Méthodes : Nous avons réalisé une étude rétrospective sur tous les enfants qui se sont présentés à un service d'urgence pédiatrique d'un hôpital universitaire sur une période d'un an. Nous avons extrait des informations de la base de données du service d'urgence. Les renseignements concernant le triage et les mesures à prendre ont été consignés dans une base de données à l'urgence par un commis après la prise en charge du patient. En l'absence d'un étalon-or pour le triage, nous avons utilisé des marqueurs de substitution de la gravité à savoir l'admission à l'hôpital, l'admission à l'unité pédiatrique de soins intensifs (UPSI) et la durée du séjour à l'urgence. La principale mesure de résultats était la corrélation entre le niveau de triage (de 1 à 5) et l'admission à I'hôpital. La corrélation entre le niveau de triage et les paramètres dichotomiques a été évaluée à
\end{abstract}

From the Division of Emergency Medicine, Department of Pediatrics, CHU Sainte-Justine, Université de Montréal, Montréal, Que.

Submitted Mar. 12, 2008; Revised June 18, 2008; Accepted Aug. 8, 2008

This article has been peer reviewed.

CJEM 2009;11(1):23-8 
l'aide d'un test du chi carré; une analyse de la variance (ANOVA) a servi à évaluer le lien entre le niveau de triage et la durée du séjour à l'urgence.

Résultats : Sur la période d'un an, 58529 enfants ont fait l'objet d'un triage à l'urgence. La proportion des enfants admis à I'hôpital était comme suit : $63 \%$ assignés au niveau de triage I (réanimation); $37 \%$, au niveau II (très urgent); $14 \%$, au niveau III (urgent); $2 \%$ au niveau IV (moins urgent); et $1 \%$ au niveau $V$ (non urgent) $(p<0,001)$. II existait également une bonne corrélation entre les niveaux de triage et la durée de séjour à l'urgence et entre l'admission à I'UPSI (les deux $p<0,001)$.

Conclusion : Cette version informatisée de l'ÉTG applicable aux enfants montre une association linéaire entre I'admission à I'hôpital, I'admission à I'UPSI et la durée de séjour à l'urgence. Ces résultats suggèrent que l'ÉTG applicable aux enfants est un outil valide pour le triage des enfants dans un service d'urgence pédiatrique.

\section{Background}

The role of triage in the emergency department (ED) is to assign priority to patients based on their need for urgent care. In other words, valid triage should assign priority to patients who need urgent care while identifying patients that may be able to wait safely. ${ }^{1,2}$ Many reports have demonstrated that overcrowding has been increasing in EDs in Canada and the United States during the past decade.$^{3-5}$ This situation highlights the importance of a valid triage system in the ED.

Different triage tools have been reported in the past decade. Among them, the Australian triage scale, ${ }^{6}$ the Manchester Triage Scale, ${ }^{7}$ the Emergency Severity Index ${ }^{8}$ and the Canadian Emergency Department Triage and Acuity Scale (CTAS) ${ }^{3}$ are the most widely used. In 2001, the Canadian Paediatric Triage and Acuity Scale (PaedCTAS) ${ }^{9}$ was developed through a consensus process and implemented in most pediatric EDs in Canada.

In the fall of 2005, a new computerized triage system (Staturg, Stat-Dev) was implemented in the ED of our institution. This is a computerized version of the PaedCTAS that uses the same wording for triage criteria. Using this tool, the nurse chooses from 38 chief complaints. Then, the computer displays the key information needed to assign the triage level in accord with the chosen complaint. This information is comprised of all the individual items from the PaedCTAS guideline that could be related to the chief complaint. Once the information is gathered, a triage level is suggested by the computer; the nurse finalizes the triage level by approving the assigned level or overriding it. The single evaluation of Staturg reported that it was associated with small improvement in the interrater reliability compared with the traditional paper-based version when applied to case scenarios. ${ }^{10}$

Only tools with proven validity should be generalized and applied to patient care. Validity is defined as the capacity of a tool to measure what it is proposed to measure. ${ }^{11} \mathrm{Be}-$ cause there is no gold standard for triage, it is difficult to properly evaluate the validity of triage tools. The "urgency" of a clinical situation can be relatively subjective. Previous studies have evaluated the validity of triage tools by comparing the triage level of live patients or case scenarios with either a panel of experts, ${ }^{10,12-15}$ or hospital admissions or resources used. ${ }^{16-19}$ These later studies have suggested that admission to hospital, length of stay (LOS) in the ED and resources use are appropriate proxy markers for patient severity.

Upon review of the medical literature, 2 small studies evaluating the validity of PaedCTAS in a clinical setting were identified. ${ }^{16,17}$ Based on this limited information, we felt it was imperative that evaluation of the validity of the PaedCTAS in a clinical setting be completed. The main objective of our study was to evaluate the correlation between different markers of severity (i.e., admission to hospital, admission to a pediatric intensive care unit [PICU] and the LOS in the ED after being seen by a physician) and the triage level assigned by nurses to children in a tertiary care hospital.

\section{Methods}

The pediatric emergency department at the Sainte-Justine Hospital assesses approximately 60000 patients annually. It is one of the 2 pediatric hospitals serving a population of 4000000 , and it has an admission rate of $8 \%$. It is a tertiary care, university-affiliated hospital. All staff physicians are residency-trained in pediatrics, emergency medicine or both. Residents and students are taught in this setting, and coverage is provided 24 hours per day.

This was a retrospective study using the computerized triage database of the ED. All patients visiting the ED for a 1-year period (between Nov. 11, 2006, and Nov. 10, 2007) were evaluated. 
During the study period, all patients presenting to the ED were triaged by a registered nurse using the Staturg triage tool. To perform triage, all nurses were required to have more than 1 year of experience in a pediatric ED, to have received appropriate training in triage and be satisfactorily evaluated by the training nurse. Staturg is a computerized version of the PaedCTAS that suggests a triage level for patients according to the information gathered by the triage nurse. The Staturg system is permitted to retrieve information regarding patient triage level, baseline demographics, time spent in the ED and outcomes. Information regarding triage and disposition was prospectively registered by the triage nurse and by a designated clerk in the ED database following each patient's management.

Because there is no gold standard for triage, surrogate markers of severity were used to evaluate the validity of the triage tool. To do so, the primary outcome measured was admission to hospital as defined by all patients for which the treating physician requested admission to hospital. There was no transfer to other facilities from our ED. Secondary outcomes were other markers of severity such as admission to the PICU, and the LOS in the ED after being seen by a physician. We used a Bonferroni correction to account for the fact that we employed multiple statistical testing; a $p$ value of 0.017 was used to signify statistical significance. To be called valid, the triage tool had to be statistically associated ( $p$ value $<0.017$ ) with all 3 markers of severity.

All data were entered in an Excel database (Microsoft Corp.) and analyzed with SPSS v16 (SPSS Inc.). The correlation between triage level and dichotomous outcomes was evaluated using a $\chi^{2}$ test. A univariate analysis of variance (ANOVA) was used to evaluate the relationship between the triage level and LOS in the ED after being seen by a physician. Triage level 3 was used a reference marker for this evaluation. The $95 \%$ confidence intervals (CIs) were reported for every result. We estimated a priori that a convenience sample of all the patients that visited the ED for a 1-year period would provide approximately 60000 patient visits. Based on the fact that the triage level with the lowest prevalence (triage 1 resuscitation) accounts for approximately $1 \%$, we expected to have at least 500 patients in each triage level.

The project was approved by the institutional review board of our institution and because of the retrospective design, no informed patient consent was deemed necessary.

\section{Results}

Over the 1-year period, 58761 patient visits were registered for the ED. They were all initially evaluated by a registered nurse whose job was dedicated to the "quick look" evaluation. Among them, 232 (0.4\%) patients left the ED after registration but before being formally triaged by a nurse and were therefore excluded from further analysis. This left 58529 patient visits to be included in the final analysis. Five patients died in the ED during the study period. They were all triaged as level 1 and were not included in further analysis because of their small number. There were no missing data for triage level, or hospital or PICU admission status. The LOS in the ED after being seen by a

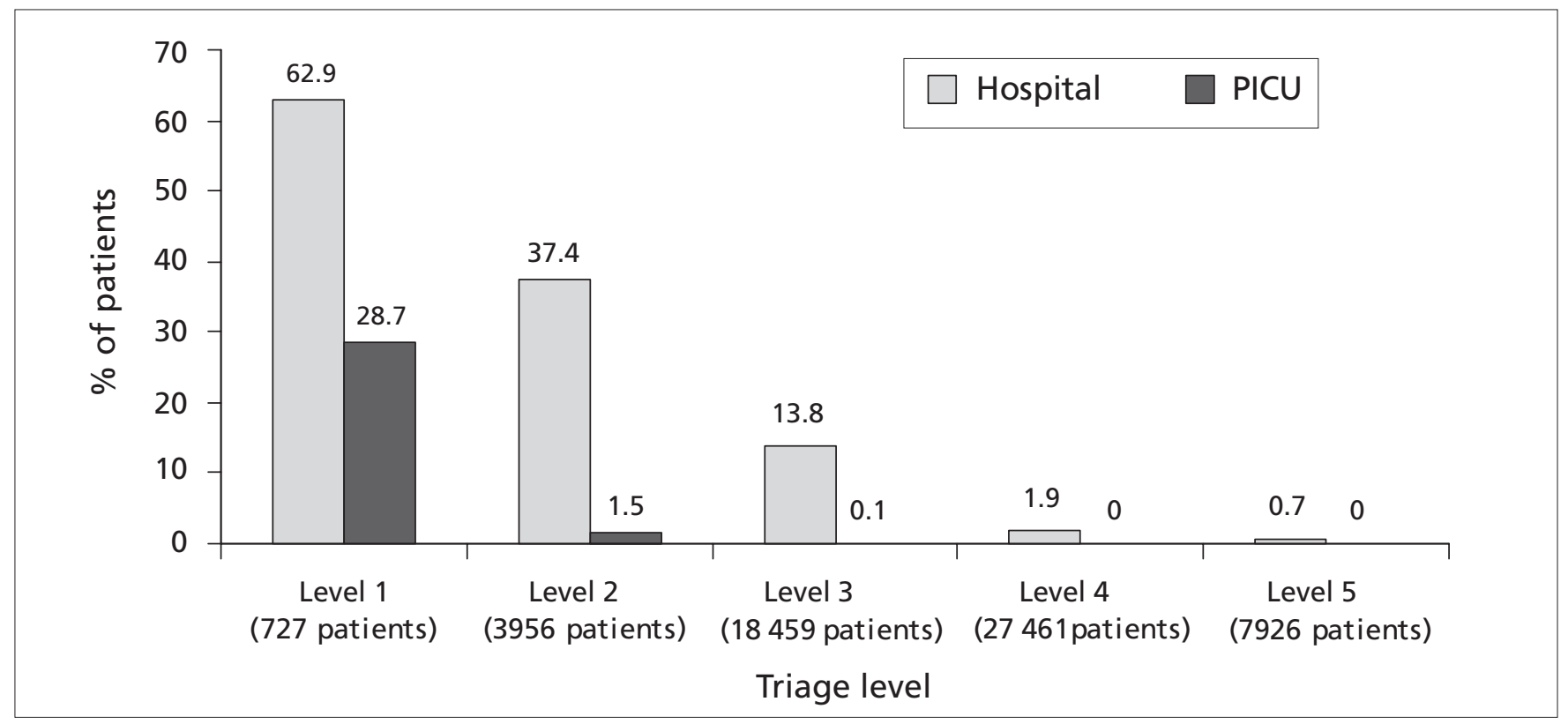

Fig. 1. Percentages of admissions to the hospital and to the pediatric intensive care unit (PICU) versus triage level $(n=58529)$. 
physician was available for the $50042(85.5 \%)$ that were seen by a physician.

Figure 1 shows that the proportion of those admitted to hospital was strongly associated with the triage level. This varied from less than $0.7 \%$ for patients triaged to level 5 , to $62.9 \%$ for patients triaged to level $1\left(\chi^{2}=9639 ; d f 4\right.$; $p<0.001)$.

Among the 5072 patients who were admitted to hospital during the study period, 298 were admitted to the PICU directly from the ED. Admission to this area was strongly associated with the triage level, as demonstrated by the fact that $90 \%$ of patients who were admitted to the PICU were

Box 1. Description of the 3 patients who were triaged to level 4 or 5 and admitted to the pediatric intensive care unit

Case

An 8-year-old boy who was triaged to level 4 for a head trauma with a normal physical exam at triage. He became drowsy after 2 hours in the waiting room and was immediately brought to the crash room for intubation. Admitted to PICU, head CT was normal.

A 3-year-old girl who was triaged to level 4 by the nurse, but was immediately brought to the exam room because she was known for neutropenia and had a fever. She should have been triaged to level 2.

An 8-year-old boy who was followed up in oncology for a bone marrow transplant for a chronic granulomatous disease. He was transferred from another hospital for an active varicella. He was triaged to level 5, but was immediately put into an isolation room in the ED. He was admitted to the PICU, because he needed to be isolated and there was no room available elsewhere in the hospital. $\mathrm{CT}$ = computed tomography; $\mathrm{ED}$ = emergency department; $\mathrm{PICU}=$ pediatric intensive care unit. initially triaged to level 1 or $2\left(\chi^{2}=11848 ; d f 4 ; p<0.001\right)$. Two patients who were triaged as semiurgent (level 4) and 1 patient who was triaged as nonurgent (level 5) were admitted directly from the ED to PICU. These cases are described in Box 1.

Figure 2 shows that a longer LOS in the ED after being seen by a physician was noted for patients with more acute triage levels. This was, however, not the case for patients triaged to level 1 (resuscitation). The ANOVA demonstrated a statistically significant association $(p<0.001)$ between triage level and mean LOS. Patients triaged to level 2 had a longer LOS than patients who were triaged to level 3 (difference: $58.4 \mathrm{~min}, 95 \%$ CI 39.5-78.4, $p<0.001$ ), patients triaged to level 4 had a shorter LOS than patients triaged to level 3 (difference: $-94.8 \mathrm{~min}, 95 \% \mathrm{CI}-76.7$ to $-113.0, p<0.001$ ) and patients triaged to level 5 had a shorter LOS than patients triaged to level 3 (difference: $-125.6 \mathrm{~min}, 95 \% \mathrm{CI}-117.9$ to $-132.9, p<0.001)$.

\section{Discussion}

Our study demonstrated that the triage level assigned by nurses using a computerized version of the PaedCTAS in our institution was strongly associated with admission to hospital, LOS in the ED after being seen by a physician and admission to PICU. Our study contributes that a novel computerized triage system was employed, a large patient sample was used and the associations identified were strong.

These results are very similar to those of Jarvis and Goldman ${ }^{17}$ who reported that hospital admission rates were

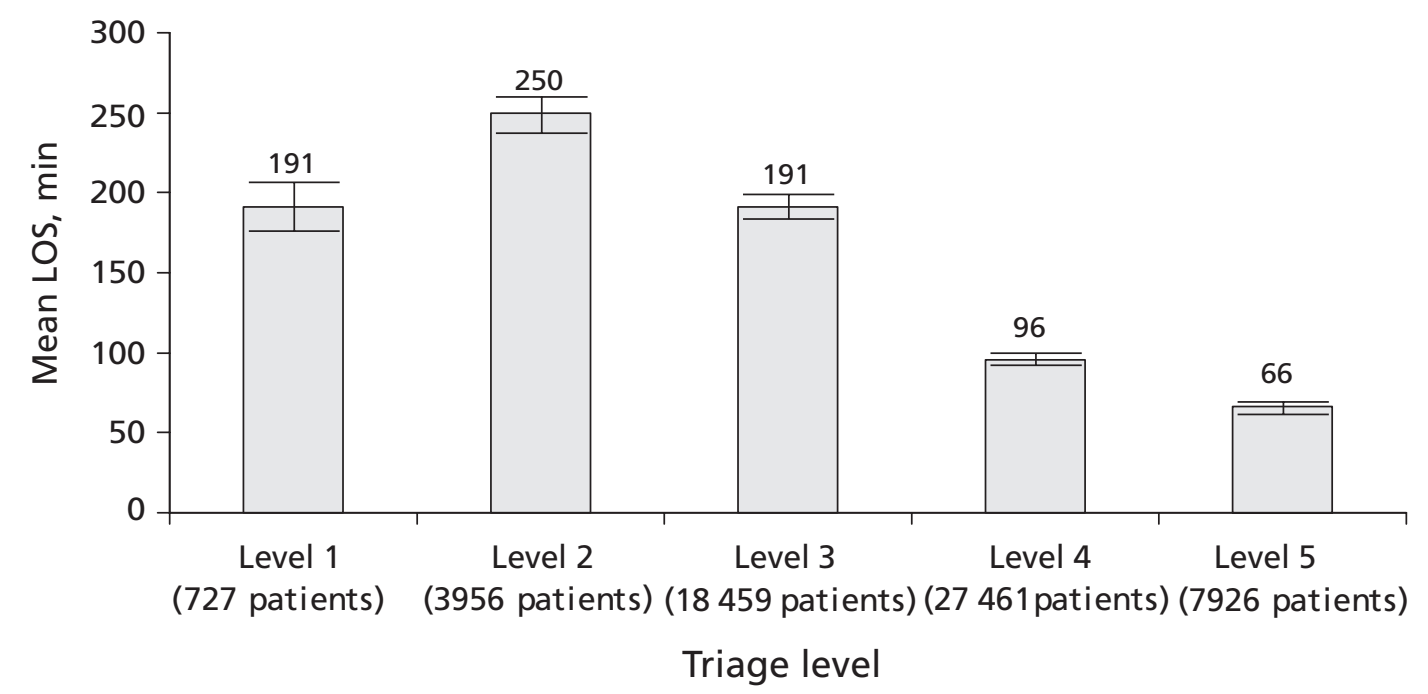

Fig. 2. The mean length of stay (LOS) in the emergency department ( $95 \%$ confidence interval) versus triage level after being seen by a physician $(n=50$ 042). 
$0 \%$ in the "nonurgent" category to $2.5 \%$ in "semiurgent," $15 \%$ in "urgent" and $45 \%$ in "resuscitation-emergent" for patients who were triaged using the PaedCTAS in their ED. ${ }^{17}$ Our results also agree with Gouin and coworkers, ${ }^{16}$ who reported the first evaluation of the PaedCTAS in a clinical setting. In this study, the authors reported hospital admission proportions of $2 \%$ for nonurgent patients, $4 \%$ for semiurgent, $12 \%$ for urgent, $24 \%$ for emergent and $100 \%$ for resuscitation among 537 children triaged using the PaedCTAS. Finally, previous studies reported agreement varying from $53 \%$ to $64 \%$ between triage nurses assigning a triage level to written case scenarios when compared with a panel of 3 experts. ${ }^{10,12,13}$

Other triage tools have also demonstrated strong association between triage levels and markers of clinical severity for children. In 2005, Baumann and Strout ${ }^{19}$ reported a high correlation between triage levels assigned by nurses using the Emergency Severity Index and hospital admission rate, resources use and LOS in the ED among 510 children visiting an ED. In 2006, Roukema and colleagues ${ }^{15}$ evaluated the ability of the Manchester Triage System (MTS) to identify true emergencies as defined by a panel of experts. Based on a sample 1065 patients, they concluded that the MTS had a sensitivity of $63 \%$ and a specificity of $78 \%$ to identify true emergencies.

A main goal of triage in the ED is to prevent a situation where a patient could deteriorate while waiting. Fortunately, these situations are uncommon in pediatric emergency medicine. Patients admitted to the PICU are usually patients with the potential to deteriorate according to the treating physician. Interestingly, there were only 2 patients triaged semiurgent and 1 triaged nonurgent who were finally admitted to the PICU from the ED. Although it may seem surprising to admit a patient triaged semi- or nonurgent to the PICU, one should note that only 3 out of the 35000 patients $(0.0086 \%)$ were triaged as semiurgent or nonurgent in our study population.

\section{Limitations}

The primary limitation of this study is based on the retrospective design. To minimize potential biases, all patients triaged during 1 complete year were included in the analysis. The information retrieved in our study was, however, mandatory for every patient registered in our ED. This explains why there were no missing data for the triage level and hospital admission rate. There were, however, some missing data in the LOS for patients that left the ED before being seen by a physician.

The fact that there is no gold standard for triage limits the strength of our conclusion. Although admission to hospital, admission to the PICU and LOS after being seen by a physician are good surrogate markers for severity, they do not encompass all urgent situations. For example, patients with isolated joint dislocation will rarely be admitted to hospital, although they are suffering severe pain and need urgent attention. These markers of severity do not take into account other reasons to increase the level of urgency such as pain or stress.

We performed our study at a single centre using a computerized version of PaedCTAS. This may limit the generalizability of the results, which would need a larger multicentre study.

Patients triaged to level 1 had a shorter LOS in the ED than patients triaged to level 2; however, this finding has been reported by others..$^{14,18}$ The reason for this observation may be related to the fact that patients admitted to the intensive care unit generally stayed a shorter time in the ED because they were admitted rapidly to the unit. These patients represented 29\% of the patients triaged to level 1.

Finally, physicians who were deciding whether to admit patients or not were not blinded to the triage level, which may have influenced the outcome. They were, however, unaware of the performance of the study.

\section{Conclusion}

Our results demonstrated that a computerized version of the PaedCTAS is strongly associated with important patient outcomes such as admission to hospital, admission to the PICU and LOS in the ED. These results suggest that PaedCTAS is highly valid for the triage of children in a pediatric ED. The validity of the PaedCTAS would need to be evaluated in multiple settings using different modalities (e.g., paper, memory and computer-based triage tools) to ensure its generalizability.

Competing interests: None declared.

\section{References}

1. McDonald L, Butterworth T, Yates DW. Triage: a literature review 1985-1993. Accid Emerg Nurs 1995;3:201-7.

2. O’Neill KA, Molczan K. Pediatric triage: a 2-tier, 5-level system in the United States. Pediatr Emerg Care 2003;19:285-90.

3. Beveridge R. CAEP issues. The Canadian Triage and Acuity Scale: a new and critical element in health care reform. Canadian Association of Emergency Physicians. J Emerg Med 1998;16: 507-11.

4. Derlet R, Richards J, Kravitz R. Frequent overcrowding in U.S. emergency departments. Acad Emerg Med 2001;8:151-5. 
5. Taylor TB. Threats to the health care safety net. Acad Emerg Med 2001;8:1080-7.

6. Fitzgerald G. Emergency department triage. Queensland (AU): University of Queensland; 1989.

7. Manchester Triage Group. Emergency triage. Plymouth (UK): BMJ Publishing Group; 1997.

8. Gilboy N, Tanabe P, Travers DA, et al. Emergency Severity Index version 4: implementation handbook. Rockville (MD): AHRQ Publication; 2005.

9. Warren DW, Jarvis A, LeBlanc L, et al. Revisions to the Canadian Triage and Acuity Scale Paediatric Guidelines (PaedCTAS). CJEM 2008;10:224-32.

10. Gravel J, Gouin S, Bailey B, et al. Reliability of a computerized version of the Pediatric Canadian Triage and Acuity Scale. Acad Emerg Med 2007;14:864-9.

11. Last JM. A dictionary of epidemiology. 4th ed. Oxford (UK): Oxford University Press; 2001.

12. Bergeron S, Gouin S, Bailey B, et al. Comparison of triage assessments among pediatric registered nurses and pediatric emergency physicians. Acad Emerg Med 2002;9:1397-401.

13. Bergeron S, Gouin S, Bailey B, et al. Agreement among pediatric health care professionals with the pediatric Canadian Triage and
Acuity Scale guidelines. Pediatr Emerg Care 2004;20:514-8.

14. Dong SL, Bullard MJ, Meurer DP, et al. Emergency triage: comparing a novel computer triage program with standard triage. Acad Emerg Med 2005;12:502-7.

15. Roukema J, Steyerberg EW, van Meurs A, et al. Validity of the Manchester Triage System in paediatric emergency care. Emerg Med J 2006;23:906-10.

16. Gouin S, Gravel J, Amre DK, et al. Evaluation of the Paediatric Canadian Triage and Acuity Scale in a pediatric ED. Am J Emerg Med 2005;23:243-7.

17. Jarvis DA, Goldman RD. Paediatric Canadian Triage and Acuity Scale as a predictor for outcome and resource utilization. CJEM 2007;9.

18. Dong SL, Bullard MJ, Meurer DP, et al. Predictive validity of a computerized emergency triage tool. Acad Emerg Med 2007;14: $16-21$.

19. Baumann MR, Strout TD. Evaluation of the Emergency Severity Index (version 3) triage algorithm in pediatric patients. Acad Emerg Med 2005;12:219-24.

Correspondence to: Dr. Jocelyn Gravel, Pediatric Emergency Department, CHU Sainte-Justine, 3175 Che. Côte-Sainte-Catherine, Montréal QC H3T 1C5; fax 514 345-2358; graveljocelyn@ @otmail.com 